\title{
Preparation of Cleaning Agent for Treatment of Abandoned Oil Pipeline
}

\author{
Fajie Yang ${ }^{l, *}$, Shengmei Chi ${ }^{2}$, Yufen Wen ${ }^{3}$, Hongtao Wang ${ }^{3}$, Jing Li ${ }^{l}$, Feng Zhu ${ }^{l}$, Chunman Li ${ }^{l}$ and Wang Tian ${ }^{l}$ \\ ${ }^{1}$ Division of security and environment, PetroChina Pipeline R\&D Center, LangFang, HeBei Province, China \\ ${ }^{2}$ Technique\&service center, Pipeline Company of PetroChina, LangFang, HeBei Province, China \\ ${ }^{3}$ Department of pipeline protection, Pipeline Company of PetroChina, LangFang, HeBei Province, China
}

\begin{abstract}
China crude oil pipeline industry commonly faces with abandonment problem because of the decreasing output of oilfield and pipeline integrity problem. China released its first standard on abandoned pipeline (SY/T 7413-2018) named specifications for the disposal of abandoned oil\&gas pipelines and it is implemented on the April 1st 2019. Pipeline cleaning is very necessary to dispose the abandoned pipeline to eliminate the risk of environment and safety. There are many cleaning agents for abandoned pipeline in the market. This chemical cleaning agent is not suitable for china pipeline because the wax remained in china pipeline is different that of foreign pipeline. The wax content in residual of china pipeline is very high. So, preparation of cleaning agent suitable for china pipeline is very important to remove wax with low cost. In this study one kind of hydrophilic cleaning agent is prepared. The result of lab test result and cleaning project both show that the kind of cleaning have excellent performance for the abandoned pipeline.
\end{abstract}

\section{Introduction}

With the increasing of the oil pipeline for the recent several decades in china, all the china pipelines on land being possessed of by PetroChina, Sinpec, Cnooc and other company exceeds twelve thousand kilometres by 2020 [1]. This pipeline infrastructure is a very important way to delivery crude oil or refinery oil. The pipeline usually links overseas markets with domestic markets, refinery factory with oil fields or customers. With the time prolonging, the oilfield company enters its middle and later periods. Some of the pipelines have to be abandoned because of the seriously decreasing output of oil field. One typical example is Daqing oil field. Water accounts for $98 \%$ the extraction from the oil well. Several pipelines connecting daqing oil field to refinery factory must be abandoned. Others pipeline have to be abandoned because of the bad pipeline integrity. The old pipelines which were built at last century are seriously corroded because all kind of compact. The abandoned oil pipeline occupied by petrochina pipeline company have exceeded 3345 kilometres by now. Some of the pipelines have been seal up for decades without cleaning procedure. The safety and environment surrounding of the pipeline is threatened by residual in the abandoned pipeline.

With the increasing of economic strength, China promulgate or modify laws to protect the environment, such as Oil and Gas Pipeline Protection Law, Environmental Protection Law, Water protection Law and soil Protection Law etc. China gets more focus on pollution issue of abandoned pipeline, especially the abandoned crude oil pipeline. China released its first China industry standard on abandoned pipeline (SY/T 7413-2018) named specifications for the disposal of abandoned oil\&gas pipelines and it is implemented on the April 1st 2019. The abandoned pipelines must be cleaned according to the standard (SY/T 7413-2018) and there must been no wax and no oil left in the pipeline [2]. The oilfield pipeline must be also disposed according to the long distance pipeline industry standard.

There are many methods for treatment on abandoned pipeline. The methods are fully developed at western developed country. They are residual cleaning, grouting, plugging and removing etc. Cleaning the residuals in the pipeline is the absolutely necessary to dispose the abandoned pipeline. The wax and oil in the pipeline can bring about risk of environment and safety. So it is mandatory step of the procedure to disposal the abandoned pipeline. Chemical cleaning method is more economical and simple than mechanical cleaning method. This is proved by pipeline company of PetroChina in 2014[3].

There are many kind of industry cleaning agents at home and abroad. These cleaning agents are not suitable for the cleaning of abandoned pipeline because the abandoned pipeline procedure is different from that of usual industry cleaning. Some foreign companies of developed country have abandoned pipeline cleaning agents. But these cleaning agents were found that it is not also suitable for cleaning domestic pipeline from the laboratory test result. This is because of the ingredient of china's crude oil is totally different from that of overseas crude oil. The content of wax and resin-asphaltenes in

\footnotetext{
* Corresponding author: doodle115@sina.com.cn
} 
china crude oil is higher than that of overseas crude oil. So the residual in the abandoned pipeline is different. It is very important to develop a kind of cleaning agent suitable for the domestic abandoned pipeline. Two kind of cleaning agent which are hydrophilic chemical cleaning Agent and hydrophobic chemical cleaning agent[4] are developed, and the result of cleaning project shows that two kinds of chemical cleaning agent have a good performance. In this paper, a new kind of chemical cleaning agent was prepared and a cleaning project was conducted to test the performance of it.

\section{Preparation of Cleaning Agent}

\subsection{Synthesise of Penetrating Agent}

Penetrating agent is a very important component in the chemical cleaning agent recipe, because it determines the performance of the cleaning agent. The speed of penetrating agent penetrate into the residual determine the dissolving speed of the cleaning agent. Firstly, a new penetrating agent was synthesized by adding reactants into the usual reactor.

\subsection{Preparation of Cleaning Agent}

Usually, a chemical cleaning agent with good performance is composed of penetrating agent, organic solvent, co-solvent, surfactant, emulsifying agent etc. when cleaning agent was injected into pipeline. The cleaning agent will act on the wax on the inner surface of the pipeline. The active penetrating agent will quickly penetrate into the wax on the inner surface of the pipeline. Then, wax will soften and peel off from the pipeline. At the help of co-solvent and surfactant, the wax will resolve in the cleaning agent. Dispersant and emulsifying agents in the cleaning agent will accelerate the above process. The wax in the pipeline will be transferred out of pipeline with the chemical cleaning fluid. Several substances are used in order to optimize the performance of cleaning agent.

The cleaning agent is prepared by using the following mixing equipment. The mixing vessel is shown by Fig.1. Penetrating agent, organic solvent, cosolvent, surfactant, emulsifying agent etc. were added to the vessel with agitating valve. The adding sequence and mixing time is very important. The speed of agitating valve can be adjusted according to the process.

\subsection{Performance Test}

\subsubsection{Dissolving speed}

In order to test dissolving speed of the cleaning agent, circle loop equipment is designed to imitate the real pipeline. Dissolving speed can be calculated by formula (1).

$$
V=h / t
$$

Where, $v$ is Dissolving speed, $\mathrm{mm} / \mathrm{min}$

$h$ is the average thickness of wax in the pipeline, $\mathrm{mm}$ $t$ is the dissolving time, min

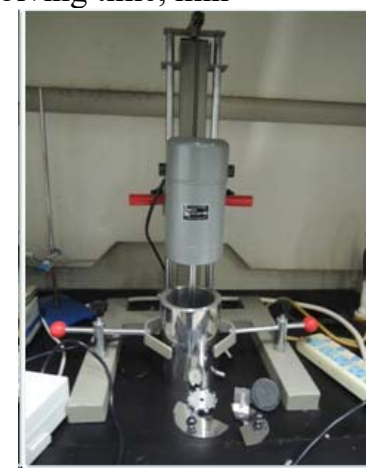

Fig.1.Mixing vessel for cleaning agent

\subsubsection{Dissolving capacity}

Dissolving capacity of the cleaning agent is a very important parameter and tested by equipment shown in the Fig.2. Dissolving capacity is calculated by formula (2).

$$
D c=m / m_{1} * 100
$$

Where, $d c$ is dissolving capacity of the cleaning agent, $\mathrm{g} / 100 \mathrm{~g}$

$m$ is the weight of wax that $100 \mathrm{~g}$ cleaning agent can dissolve

$m_{l}$ is the weight of cleaning agent.

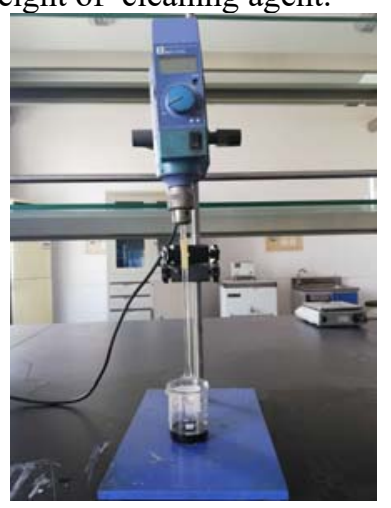

Fig.2. dissolving capacity test equipment

It can be found that there is an agitating valve attached to test equipment for dissolving capacity. The speed of agitating valve is very important. The speed of blender should not too fast. This is because when cleaning pipeline the advance speed of cleaning pig should be as low as possible. The cleaning agent has a longer time to contact with the wax.

\section{Results and Discussion}

\subsection{Synthesise of Penetrating Agent}

In order to synthesize penetrating agent with good performance, many substances was used. And the rests show 1-chlorobutane and cinene are good option. The effect of reaction temperature, time and accelerating agent on the performance of cleaning agent was investigated. 


\subsubsection{Effect of Reaction Accelerating Agent}

The reaction between 1-chlorobutane and cinene is low. Accelerating agent was used to accelerate the reaction. Three kind of accelerating agent were used to investigate the effect on the performance of cleaning agent. The result is shown in Fig.3. From the Fig.3, it can be seen that $b$ type accelerating agent have best accelerating performance.

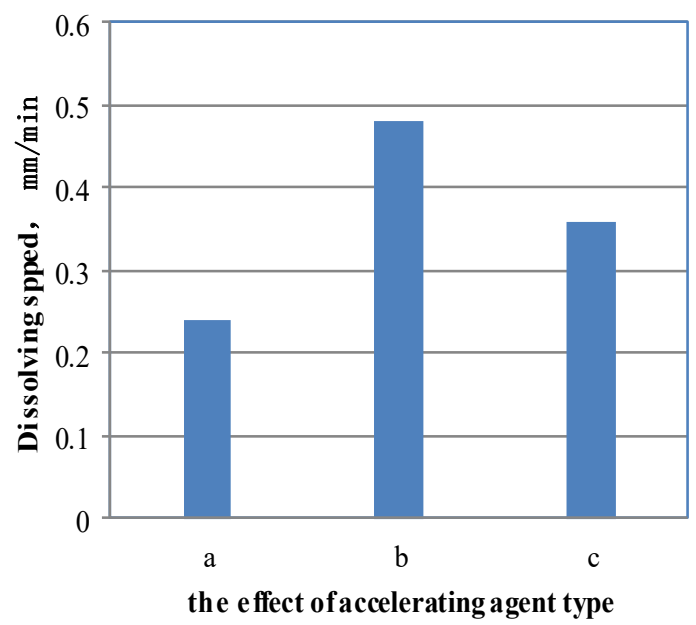

Fig.3. the effect of accelerating agent type on the cleaning performance

\subsubsection{Effect of Reaction Temperature}

In order to study the reaction temperature on the performance of the cleaning agent, six reaction temperature $25,50,75,100,125$ and $150{ }^{\circ} \mathrm{C}$ were selected. The result is shown in Fig. 4 . It can be seen that the dissolving speed increases with the increasing of the reaction temperature. When the reaction temperature is higher than $100^{\circ} \mathrm{C}$, the dissolving speed keep almost constantly.

\subsubsection{Effect of Reaction Time}

Lastly, effect of reaction time on the performance of cleaning agent is study. Before 1-chlorobutane and cinene are initiated the $b$ type accelerating agent is added into the reaction system. The test result is shown in Fig.5. From the Fig. 5 it can be seen that $120 \mathrm{~min}$ reaction time is enough.

\subsection{Preparation of cleaning agent}

In this study, a kind of hydrophilic chemical cleaning agent is prepared. It is well known that hydrophilic chemical cleaning agent is a kind of water soluble agent. Hydrophilic cleaning includes penetrating agent, organic solvent, co-solvent, surfactant, emulsifying agent etc. Firstly, the penetrating agent fast penetrates into the wax and let the wax peel off from the pipeline. At the help of co-solvent and surfactant, the wax will resolve in the cleaning agent. Dispersant and emulsifying agents can decrease surface tension between cleaning agent and wax, and it will accelerate the wax resolving process. Co-solvent is a kind of organic which can assist the residual in the inner surface of abandoned pipeline to solve in the cleaning agent. The organic with high dissolving speed for wax is usually chosen as solvent or co-solvent, such as alcohols, ether, esters, alkane etc. the type of organic solvent and co-solvent determined the dissolving speed of the chemical cleaning agent. Toxic substance is not allowed to be added into the chemical cleaning agent, because toxic substance is harmful for human being, the soil, groundwater and surface water. It is harmful to the environment. Usually, small molecular organic compound was selected as penetrating agent because it can easily penetrate into the wax.

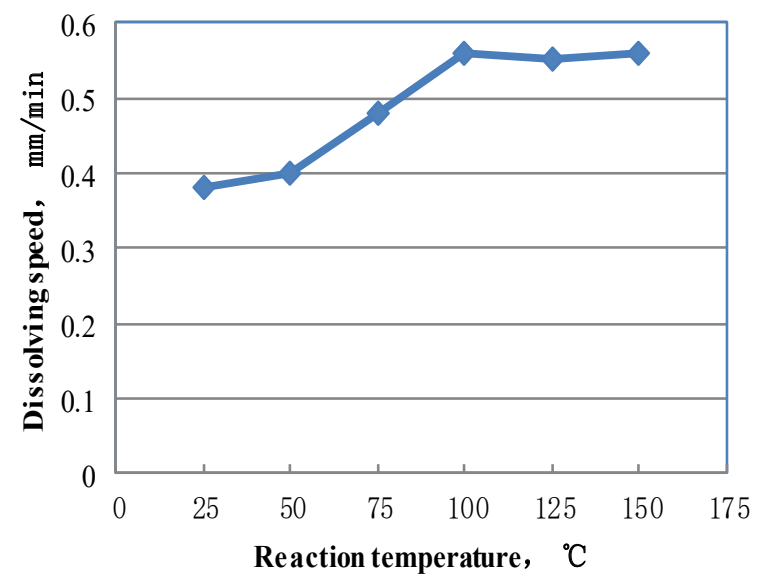

Fig.4. the effect of reaction temperature on the cleaning performance

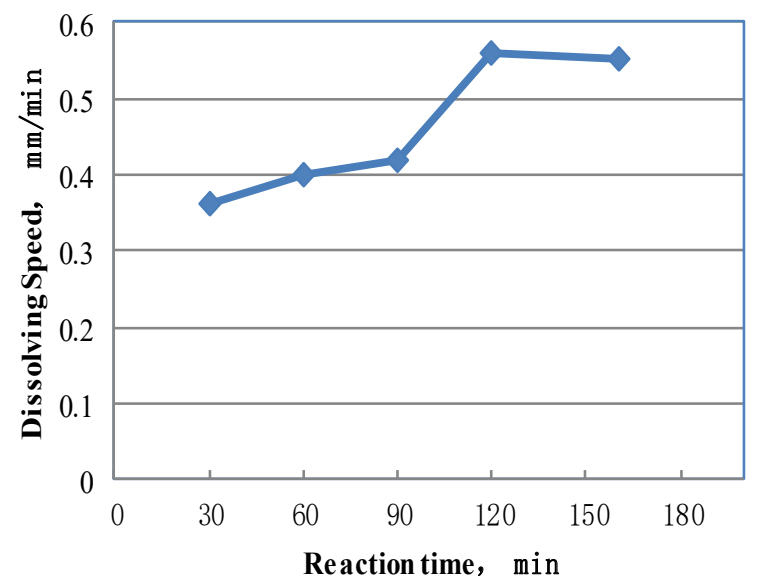

Fig.5. the effect of reaction time on the cleaning performance

Surfactant is necessary ingredient of chemical cleaning agent. It can reduce the surface tension of the solid-liquid surface. In the cleaning process of abandoned pipeline it can reduce the surface tension between wax and cleaning agent. All kinds of surfactant could be used in it, such as the ordinarily nonionic, anionic and cationic surfactant. It also can increase the dissolving capacity of cleaning agent. Furthermore, it also can increase dissolving speed of wax in cleaning agent. When penetrating agent penetrates into wax, the wax will soften and peel off from the pipeline. The dispersant can assist the cleaning agent transfer residual 
outside the abandoned pipeline with cleaning agent fluid. It is important to the cleaning performance and capacity.

In order to prepare cleaning agent cooperation with the synthesized penetrating agent, many tests were conducted to determine the type of co-solvent, dispersant and surfactant. It is found that it is good selecting isooctanol as co-solvent, FFA (alkylolamides) as dispersant, SDBS (sodium dodecyl benzene sulfonate) as surfactant. L9 $\left(3^{4}\right)$ orthogonal test was conducted to further conducted to determine the concentration of cosolvent, dispersant and surfactant. A factor is SDBS, and factor level is $0.8 \%, 1.6 \%$ and $2.4 \%$. B factor is synthsized penetrating agent, and factor level is $20 \%$ 、 $30 \%$ and $40 \%$. C factor is isooctanol, and factor level is $2 \%, 4 \%$ and $8 \%$. D factor is FFA, and factor level is $0.2 \%, 0.5 \%$ and $0.8 \%$.

$\mathrm{K}$ is calculated by the orthogonal test result and listed in the table 1. it can be seen that A2、B3、C2 and D3 is the optimized factor level. The importance list of effect on the cleaning agent performance is $\mathrm{B}>\mathrm{D}>\mathrm{C}>\mathrm{A}$. so the optimized concentration is $1.6 \%, 40 \%, 4 \%, 0.8 \%$, SDBS, penetrating agent, isooctanol, FFA respectively. The rest is water. The dissolving speed of the cleaning agent is $0.56 \mathrm{~mm} / \mathrm{min}$, and the capacity is $1.1 \mathrm{~g} / \mathrm{g}$.

Table 1. L9 $\left(3^{4}\right)$ test result and analysis

\begin{tabular}{|c|c|c|c|c|c|}
\hline \multirow{2}{*}{$\begin{array}{l}\text { Test } \\
\text { No }\end{array}$} & \multicolumn{4}{|c|}{ factor } & \multirow[b]{2}{*}{ DS } \\
\hline & A & B & $\mathrm{C}$ & D & \\
\hline 1 & 1 & 1 & 1 & 1 & 0.32 \\
\hline 2 & 1 & 2 & 2 & 2 & 0.48 \\
\hline 3 & 1 & 3 & 3 & 3 & 0.56 \\
\hline 4 & 2 & 1 & 2 & 3 & 0.4 \\
\hline 5 & 2 & 2 & 3 & 1 & 0.45 \\
\hline 6 & 2 & 3 & 1 & 2 & 0.55 \\
\hline 7 & 3 & 1 & 3 & 2 & 0.31 \\
\hline 8 & 3 & 2 & 1 & 3 & 0.47 \\
\hline 9 & 3 & 3 & 2 & 1 & 0.54 \\
\hline K1 & 1.36 & 1.03 & 1.34 & 1.31 & \\
\hline K2 & 1.4 & 1.4 & 1.42 & 1. 34 & \\
\hline K3 & 1.32 & 1.65 & 1. 32 & 1.43 & \\
\hline k1 & 0.453333 & 0.343333 & 0.446667 & 0.436667 & \\
\hline k2 & 0.466667 & 0.466667 & 0.473333 & 0.446667 & \\
\hline k3 & 0.44 & 0.55 & 0.44 & 0.476667 & \\
\hline $\mathrm{R}$ & 0.026667 & 0.206667 & 0.033333 & 0.04 & \\
\hline List & & $B>D$ & $\mathrm{C}>\mathrm{A}$ & & \\
\hline level & A2 & B3 & $\mathrm{C} 2$ & D3 & \\
\hline & & A2 & 2D3 & & \\
\hline
\end{tabular}

Good stability is a very important factor for a cleaning agent. In order to test its long term storage stability performance a cleaning agent sample was put in the lab for more than two month. It is very uniform and stable. The longer time stability performance will be continues to observe.

5 tons chemical cleaning agent was prepared using SDBS, self-synthsized penetrating agent, isooctanol,
FFA. Their concentrations are $1.6 \%, 40 \%, 4 \%, 0.8 \%$, respectively. This chemical cleaning agent was used to clean oil pipeline belong to the PetroChina in the qinhuangdao. One kilometre pipeline were divided from abandoned pipeline which total pipeline length is 306 kilometres. Thickness of the wax left in the pipeline is about $206 \mathrm{~mm}$. It can be seen from a picture of the Figure 6. The pressure test shows that integrity of the pipeline is very good. Launching chamber and receiving chamber was installed at the two end of pipeline. Firstly, polyurethane pig was launched. Then, straight plate pigs with different interference were launched. Wax was collected and analysed. The dissolving speed of cleaning agent was calculated. The advance speed of pig was determined by dissolving speed. The cleaning project was made according to the laboratory and calculating result. After seven days' work, the divided pipeline was cleaned. The cleaned inner surface is showed in the $b$ picture of Fig.6. It is totally clean to meet the demand of the standard (SY/T 7413-2018).

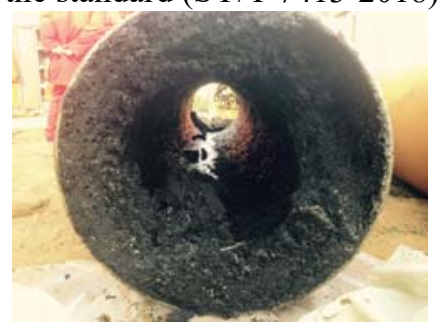

a. before cleaning

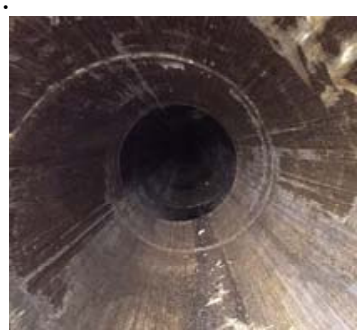

b. after cleaning
Fig.6. Effect of hydrophilic cleaning agent

From the figure, it can be seen that the self-prepared cleaning agent has very excellent cleaning performance. The cleaned pipeline can meet the demand of the Pipeline Company and Liaoning local government. Because the pipeline is located in the farmland, it was directly abandoned in place.

\section{Conclusions}

Pipeline cleaning is the necessary step to dispose the abandoned pipeline because it can eliminate the risk of environment and safety. One kind of hydrophilic chemical cleaning agent is prepared and it shows excellent performance in lab and cleaning project. It will be used to clean more abandoned crude oil pipeline.

\section{References}

1. T. Wang, X. Wang, L. Z. Rong, L.N. Xue, Z. B. Gao, T. W. Wang, J. Oil \& Gas stor. and trans., 36, 11 (2017)

2. H. T.Wang, Y. W. Kang, Z. G. Liu, specifications for the disposal of abandoned oil\&gas pipeline,2018

3. H.T. Wang, F. J. Yang, R. G. Li, ICAEER 53, 04040(2018)

4. F. J. Yang, C. M. Li, J. Li, H.T. Wang, ICAEER 118, 04025(2019) 